\title{
Application to the additive fabrication of Object Oriented Methodology
}

\author{
Jean-François Couturier ${ }^{1}$ and Alexandre Schneider ${ }^{1 a}$ \\ ${ }^{1}$ Reims University FRANCE - CReSTIC - NUM3D AM platform
}

\begin{abstract}
Based on some experimentation in NUM3D Platform, we integrate the possibilities of additive manufacturing processes and the characteristics of the materials. We extend to the specific property inherent in form and control to define a new tetraptych CPMF (Control/Process/Material/Form). We propose, from this tetraptych, an Oriented Object Modelization suited to Additive Manufacturing. In this paper, we set up a virtual representation specific to industrial manufacturing. The final objective, in fine, will be to use this representation in a decision-support tool.
\end{abstract}

\section{Introduction}

The objective of this paper is to produce an Object Oriented Methodology like structure suited to Additive Manufacturing (AM). Ultimately, we will use these attributes to implement a decision support tool. The result will be a numerical model integrating all the manufacturing constraints.

Our Object Oriented model integrate the manufacturing knowledge. In particular, the possibilities of manufacturing processes but also the characteristics of the materials and the ability to control the parts produced.

The proposed structure will permit to better take into account the specificity of additive manufacturing in numerical simulation algorithms (As meso-structures or bio-mimicry). That will also be able to validate and define an artifact part for validating a process or directly a machine.

The establishment of a detailed ontology of additive manufacturing was required to clearly explain the parameters who are, for time, implied or empirical. This ontology has two objectives, allow experienced users to better understand the field, and for novices, to get the ability to integrate this technology in their specialties (e.g. topology optimization).

\section{State of art}

\footnotetext{
${ }^{a}$ Corresponding author: alexandre.schneider@univ-reims.fr
}

\subsection{Database}

There are many specific database to materials as well as processes but few that combine the two. Marsden [10,11] highlights the role of metadata in manufacturing by integrating processes and material properties with the world of product development. Munguia [12], meanwhile, shows how to integrate a database into an artificial intelligence system dedicated to additive manufacturing.

Based on the work of Professor Ashby [13], the company GRANTA Design is the leading materials information management system. With their solution GRANTA MI "you can apply this proven software to capture all of your vital data on Additive Manufacturing. Data is captured in one place, with full traceability".

The objective is to create an essential knowledge base to develop a better understanding of processes (especially additive manufacturing). This database must be expanding by integrating other essential criteria in order to serve as a tool for qualification and certification of manufactured parts.

Additive Manufacturing Template Database provides a data structure (Figure 1) developed by Granta based on extensive experience from AMAZE projects. 


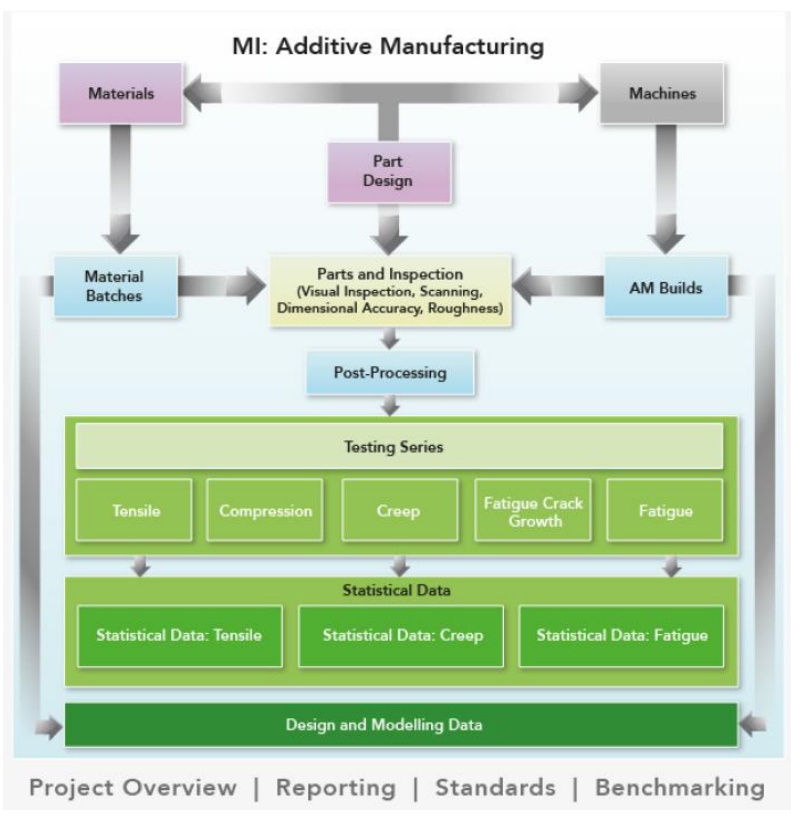

Figure 1. GRANTA AM Schema

It allows the integration of data from our own laboratory test or based on our experience, like:

- "Import 'logfiles' directly from Additive Manufacturing machines, store process parameters, extract logged data for specific builds

- Link build information directly to supplier data on the batches of material that were processed to make a part

- Capture and associate part finishing, inspection, and test data with individual parts, and feed these results into statistical analyses to determine mechanical properties and monitor reproducibility

- Export properties for use in simulation and design, capturing simulated outputs for use in optimizing part design and production."

\subsection{Object Oriented Modelling}

The object-orientation is a well know technique in informatics. This technique was introduce in 1966 by OleJohan Dahl and Kristen Nygaard [8], and was developed in the works of Alan Kay in 1968 [9]. For this work, they respectively win the Turing price in 2001 and 2003.

The main idea of object-orientation is "don't care how the others part of the system make for obtain the answer of your question, just have an answer". Object are see like some "black-box", only the public properties are in interest. Habitually, the language used for represent object is UML. The model abstracts the reality in some virtual object who interact each other. The real world is built from material or immaterial object, who are represent in the model.

The Object orientation is in fact a technique for broach a problem and cut it in smaller sub-problem.

The originality of our proposal is to use the OOM to virtualize the characteristics inherent in manufacturing production and in particular to additive manufacturing. A priori, we do not know any works developed in this sense.

\subsection{Ontology in Additive Manufacturing}

An Oriented Object model is a theoretical construction translating a practical case. The additive manufacturing is a new technology and, therefore, don't have the necessary objectivity for formalize this practice. We will base on the existent norm ISO CD/17296-2 that we reinforce in this paper with an AM dedicated ontology.

An ontology defines a common vocabulary for researchers who need to share information in a domain. [1] [2] [3-7]

- Explain what is considered implicit in a domain (e.g. AM)

- Distinguish field knowledge with respect to operational knowledge

\section{Problem}

Our goal is to link the operational properties and structural properties of classes modeling the constraints inherent in manufacturing production, particularly in the context of additive manufacturing.

All existing databases is based primarily on the characteristics of processes and materials.

However, and by feedback from multiple test on NUM3D Platform, we consider that we need to more characteristics.

Therefore, we propose two new characteristics (Control and Form) and define thus our "tetraptych CPMF" (detailed in Figure 0).

\subsection{Generating a software}

For a given part (forms / materials), we would like to develop a decision-making tool for the manufacturing process. As part of our work, we have a focus on additive manufacturing but the proposed methodology is applicable to other "traditional" processes.

Implementing a basic algorithm, that can adapt to the different elements of our "tetraptych CPMF" (Control / Process / Materials / Form) defining a manufactured object, requires a dynamic evolution of the program. Depending on the differences of each element of our tetraptych, the responses to the same need may require different methods. For example, for the realization of the part below (Figure 2) our need is to prevent the collapse of the cantilever. The provided method differs depending on the type of process, so the generation of a same support material is necessary in FDM, a filling with a support material in Material Jetting process but requires no action Binder Jetting SLS because the powder bed provides this function. 


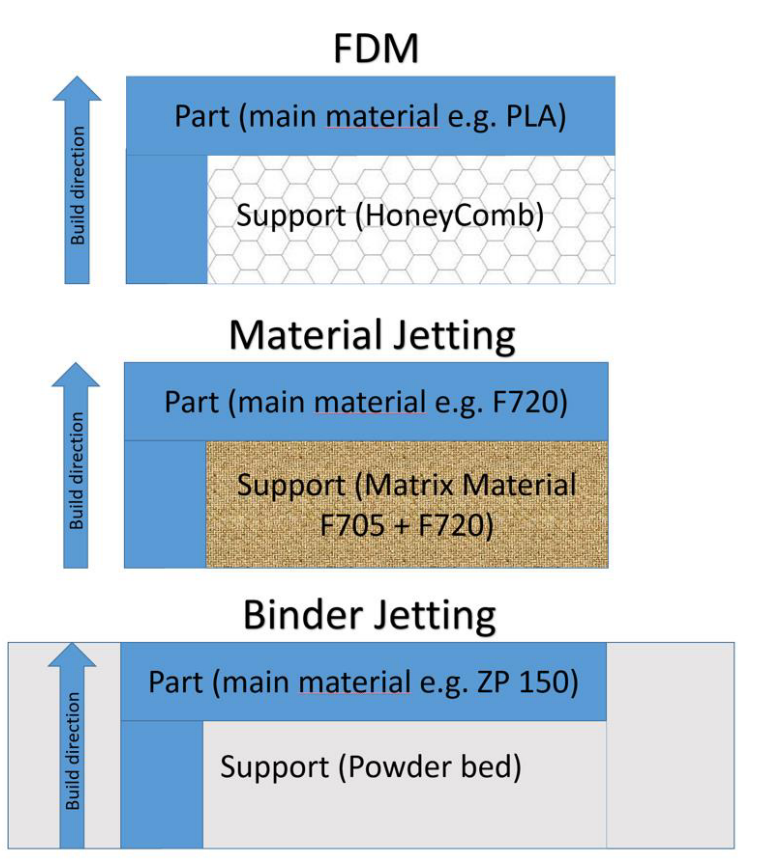

Figure 2. Part with cantilever

In principle, object programming is based on the existence of classes implementing different need for a more comprehensive algorithm. A class is defined virtually, via the methods as to answer a specific question, and this in "black box".

Due to the concept of heritage, it is possible to implement the same abstract class in several different ways, thus adapting dynamically to changing the algorithm based on internal or external parameters in the program.

It is for the reasons that the object-oriented methodology seemed appropriate to resolve our problem.

The same structure will also specify existing algorithms.

For example, topology optimization algorithms are not specifically adapted to additive manufacturing but are general. Our proposal will guide the seven standardized additive manufacturing processes and thus to best adapt the numerical models to mechanical stress in their environment. Work in support of the project OptiFabAdd allow to highlight a marked improvement forms topologically optimized by adding own specificities to AM $[17,18]$.

Topological optimization algorithms are of course not the only ones to be adapted to additive manufacturing. Indeed, many studies concerned with meso-structures as well as mimicry [19], [20], [21], [22], [23], [24]. A development suited to additive manufacturing would specialize the algorithms and make them more efficient.

\subsection{Consideration of dimensional control / geometric / topological}

The vast majority of work who linked, by a general database system, the materials to processes do not take into account the ability of the process to realize dimensionally the parts (accuracy). Therefore, we propose the integration of a criteria "Control" able to take in to account the needed of accuracy and check the faculty of the device. There is no need to design a better part that the device can realize or that we can control!

The Faculty of additive manufacturing device make freeform forced us to integrate 3D scanning means $[25,26]$ in addition to the "traditional" control means such as CMM [27].

The resulting program will allow users a decision support at various optimality parameters depending on our tetraptych. Thus a requirement of a given object, we can propose the better process (among the seven standardized additive manufacturing process) depending on the needs.

For example, a requirement of precision and a need for rapid manufacturing, the software will return us the fastest process capable of satisfying the required accuracy and associate the appropriate means of control. The result of software will give not only the method but also the associate devices.

\subsection{Integration of knowledge in processes and specific materials for additive manufacturing}

To validate your model, we based on the capitalization of knowledge realized on the NUM3D platform (CReSTIC laboratory from University of Reims Champagne Ardenne). We were able to realize different test allows us to determine the evolution in the mechanical characteristics in function of three criteria (materials, processes and orientation) on machines at our disposal [25]. The establishment DOE has given us the critical values on the limits of our machines. Thus, the minimum wall thickness or the minimum diameter of printable and cleaned without deterioration channels of the parts could be determined according to the materials and processes used in manufacturing [15].

\section{Proposal for structuration}

We wish to enrich and complete this type of bases by adding two new characteristics:

- The shape (this notion being just begun in some bases...): The geometric analysis of a 3D model allowing us, for example, fixed materials and processes, to predict differences in resistance of the part manufactured. It also allows, according to the methods, to define a better orientation of the workpiece manufactured in the device environment.

- Means of controls: we wish to integrate earlier in the manufacturing process, the possible constraints inherent to input controls. It is also important to align the precision of the manufacturing and control tools.

These two new characteristics (Shape and Control) added to conventional constraints (Process and Material) 
define what we call the "tetraptych CPMF" (Control / Process / Materials / Form).

\subsection{Classes using tree}

According to [6], we have detailed our proposition into 6 key steps:

- Definition of the Domain and its scope;

- Reuse of existing ontologies; (e.g. CES)

- Enumerate important terms in the ontology; (e.g. Brainstorming, FreeMind)

- Definition of classes and their prioritization;

- Properties of classes and attributes;

- Creating instances;

The manufacturing processes are all types (subtractive / additive / other). In our study, we have focused on the additive manufacturing processes.

\subsection{Description of structuration}

Our goal is to analyze a manufactured product split into four independent aspects. These aspects are (figure 3):

- The shape of the object and all its geometric characteristics modeled in 3D: class = "Form"

- The constituent materials with their mechanical characteristics: class = "Materials"

- The manufacturing processes have realized the piece manufactured with all these specific: class = "Process"

- A method (or more) to verify the compliance of the finished product: class = "Control"

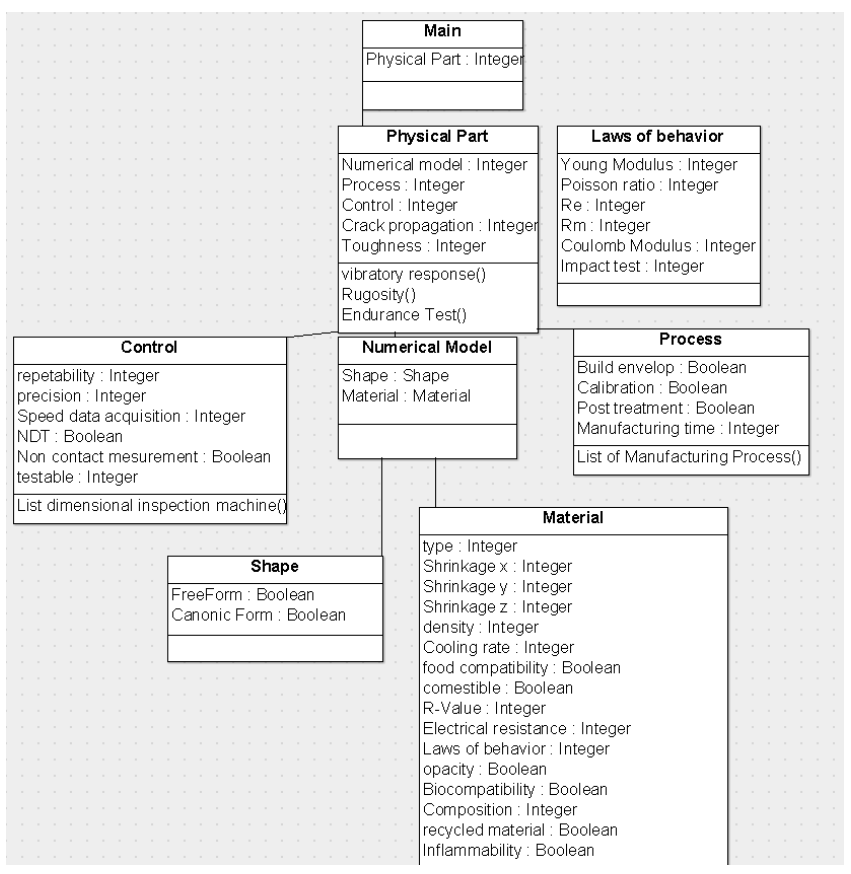

Figure 3. Object Tree

In the CAD modeling (CATIA, SW, PTC ...), we can numerically represent a product based on its shape and constituent material. Therefore, we call "numerical model" a class composed by the two classes "Shapes" and "materials" and which will represent the product DFN.

In fact, we imply that it is not usually to take into account the means of control or implementation when defining 3D modeling. Although many studies demonstrate the importance of this integration (Concept DFM) [28]

The finished manufactured product will be represented by the class "physical part" composed by classes "Process" - "Control" - "Numerical Model".

The "numerical model" based itself on classes "Shape" and "Material"; our physical part consists of classes that represented the four independent aspects of our structure.

The integration of our proposal at the heart of FEM software requires a lot of questioning of the laws of behavior. For easier using, we have chosen to group the "Laws of behavior" in a separate class who can easily be given as a parameter to specific programs.

\subsection{Classes and heritage}

One of the main objectives of our modeling is to allow dynamic adaptation of the calculation and optimization algorithms based on the consideration of criteria and parameters depending on the choice made on our tetraptych. The calculations of theoretical mechanical resistance, the forecast cost of a manufactured part or the production time, are intimately linked to the choices that have been made on the tetraptych (change materials, shape, manufacturing process or control).

The classes described in your model are abstract, and destined to be inherited according to the different opportunities available to each element of tetraptych. As in a conventional object modeling, the highest class in the interaction diagram are able to query their classes that are less based on attributes and predefined functions abstract classes. When a class is questioned, it behaves like a "black box" and a change in method or formula can be easily performed by inheriting inter-changing different classes of the same abstract class.

For example, we will focus on the abstract class "Process" of manufacturing. We know from the abstract class, a "process" must be able, among other things, an estimate of the volume of work available, or the time required to manufacture a part size and given shape. The manufacturing process should also be able to provide a list of its own characteristics to influence the strength of a part, or be able to validate the feasibility of a given digital model.

We consider as a first inheritance step does distinguish between different categories of manufacturing process, defining three new abstract classes inheriting the class manufacturing process:

- $\quad$ Subtractive manufacturing

- Additive manufacturing

- Other processes 
Our study is focused on additive manufacturing (subtractive manufacturing and other process were not further).

As for additive manufacturing, we then describe concrete seven classes, based on the seven additive manufacturing processes as defined in the standard "Additive manufacturing General principles Part 2: Overview of the categories of processes and basic materials" [27].

Each of these classes must be able to answer any questions that may be asked in a manufacturing process. But each will have different function and method to answer these same questions, in fact inducing a dynamic modification effect of the global algorithm depending on the chosen manufacturing process, and therefore the instantiated class to represent it.

\section{Opening}

Our proposal aims to formalize the characteristics of our tetraptych bases in an abstract modeling to be completed and implemented in order to specialize in digital algorithms uses in engineering.

For example, and as an opening to our work, we search to adapt to the context of AM a topological optimization, bio-mimicry or meso-structures generation algorithms in the heart of our manufactured parts.

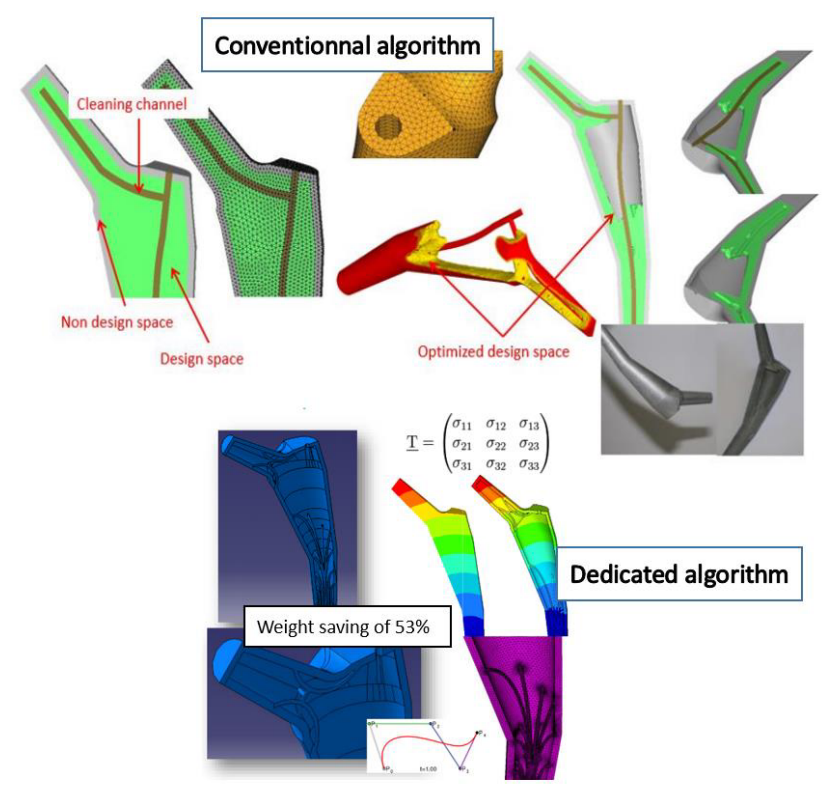

Figure 4 . Application OptiFabAdd $\odot$ Project

Within the OptiFabAddC industrial project, we were able to show that adaptations of generic algorithms (here topological optimization) by integrating the inherent parameters in the manufacturing used process (Figure 4), allow a significant gain in expectations (This dedicated optimization material allows savings of 53 per cent in terms of weight compared to a conventional topological optimization only 47 per cent).

Beyond this application, taking into account any changes in the tetraptych bases "CPMF" is likely to bring significantly improved responses in generic algorithms mentioned above.

This topological optimization application shows that taking into account inherent specificity of Additive Manufacturing generate convincing results. In OptiFabAddC, this application was not automatized and could be easily due to our model.

\section{Conclusion}

This paper proposes an Object Oriented Methodology structure specifically adapted to additive manufacturing technologies. We propose a tree called "CPMF" (Control / Process / Material / Form) for, among other things, provide a dynamic response to various parameters of a tetraptych for conventional simulation algorithms (i.e. topology optimization) and dedicated to the AM.

The first results through OptiFabAdd(C) for topological optimization applied to the AM are promising and encourage us to expand our work for meso-structures and bio-mimicry like algorithms.

\section{Acknowledgment}

This project is partly based on the NUM3D platform (partners: UTT, ENSAM and CReSTIC for URCA). We also thank the Research Center DINCCS for OptifabAddC Project. Advices on Topological Optimization from Nicolas Gardan CAD/CAE deserves our heartfelt thanks.

\section{References}

1. CW Holsapple, KD Joshi. Communications of the ACM, 45:42-7 (2002).

2. $\mathrm{D} \mathrm{Wu}, \mathrm{A}$ Håkansson. Procedia Computer Science, 35, 504-13 (2014).

3. $\mathrm{R}$ De Almeida Falbo, $\mathrm{G}$ Guizzardi, KC Duarte, Proceedings of the 14th international conference on Software engineering and knowledge engineering, ACM, 8, p. 351 (2002).

4. R De Almeida Falbo, CS de Menezes, ARC da Rocha. Progress in Artificial Intelligence-IBERAMIA 98, Springer, 60 p. 349 (1998).

5. F Ameri, C Urbanovsky, C McArthur, Proc. 7th International Conference on FOIS 2012, Graz, Austria, Citeseer (2012).

6. NF Noy, DL McGuinness, and al., Stanford knowledge systems laboratory technical report KSL-01-05 and Stanford medical informatics technical report SMI2001-0880 2001).

7. S Staab, R Studer, H-P Schnurr, Y Sure, IEEE Intelligent Systems 16 26-34 (2001). 
8. O-J Dahl, K Nygaard Communications of the ACM 9 , 8671 (1966).

9. A Kay Unpublished Master's Thesis, University of Utah, Salt Lake City, UT, United States (1968).

10. W Marsden, S Warde, E Cope, D Debon 56th AIAA/ASCE/AHS/ASC Structures, Structural Dynamics, and Materials Conference, American Institute of Aeronautics and Astronautics;

11. W Marsden, S Warde Granta Technical Paper (2010).

12. J Munguía, J Lloveras, S Llorens, T Laoui International Journal of Production Research 48 2261-78 (2010).

13. GRANTA M. Granta Design Ltd. Cambridge, UK, www Grantadesign/products/mi

14. N Gardan International Journal of Manufacturing Engineering (2014).

15. N Gardan, A Schneider Journal of Manufacturing Systems.

16. CB Williams, JK Cochran, DW Rosen Int J Adv Manuf Technol 53 231-9 (2010).

17. M Vesenjak Polymer Testing 29 991-4 (2010).
18. W Miller, CW Smith, F Scarpa, KE Evans Composites Science and Technology 70 1049-56 (2010).

19. Y Sugimura Mechanics of Materials 36 715-21 (2004).

20. D Prall, RS Lakes. International Journal of Mechanical Sciences 39 305-14 (1997).

21. H Abramovitch, M Burgard, L Edery-Azulay, KE Evans, M Hoffmeister, W Miller, et al. Composites Science and Technology 70 1072-9 (2010).

22. BR Barbero, ES Ureta Computer-Aided Design 43 188206 (2011).

23. A Schneider, JM Coulon, F Boyer, CB Machado, N Gardan, N Dey, et al. IEEE Conference: Computing for Sustainable Global Development (2015).

24. J Kroll, S Botta, J Breuninger, A Verl vol. 8650, p. 11 (2013).

25. A Schneider, N Gardan, J Gardan 21ème CFM (2013).

26. K Swift, N Brown, Journal of Engineering Manufactured vol.217 827-33 (2003).

27. UNM920. Fabrication additive Principes généraux Partie 2: vue d'ensemble des catégories de procédés et des matériaux de base (2015). 